population. DNA was extracted from whole blood and Guthrie card specimens. Surfactant single nucleotide polymorphism (SNP) genotyping was arranged using the Taqman technique. ACE gene primers were obtained for the previously known insertion/deletion polymorphism and PCR analysis was performed.

Results 236 infants born prematurely survived to 28 days postnatal age and contributed to genotype analysis. 106 infants did not develop BPD and 130 infants did develop BPD (54 mild, 29 moderate, 47 severe). Both gestational age and birth weight were significantly different between those infants who did and did not develop BPD and predicted BPD development with an area under the ROC of 0.88 and 0.82 respectively. We demonstrated using multifactorial statistical analysis that the inclusion of the ACE genotype to a predictive statistical model of BPD development improves the predictive potential of the model (area under ROC curve 0.88).

Conclusion The presence of the ACE DD genotype is associated with a higher likelihood of developing BPD.

\section{PS-209 BCG VACCINATION CAN PREVENT HYPEROXIC LUNG INJURY?}

${ }^{1} \mathrm{~A}$ Kumral, ${ }^{1} \mathrm{~B}$ Iscan, ${ }^{2} \mathrm{~S}$ Micili, ${ }^{1} \mathrm{~F}$ Tuzun, ${ }^{1} \mathrm{~N}$ Duman, ${ }^{1} \mathrm{M}$ Arslan, ${ }^{2} \mathrm{~K}$ Tugyan, ${ }^{1} \mathrm{H}$ Ozkan. ${ }^{1}$ Pediatrics Neonatology, Dokuz Eylul University Faculty of Medicine, Izmir, Turkey; ${ }^{2}$ Histology and Embryology, Dokuz Eylul University Faculty of Medicine, Izmir, Turkey

\subsection{6/archdischild-2014-307384.507}

Introduction The aim of this study effects of BCG vaccine on the histopathologic and gene expression changes seen in hyperoxia induced neonatal rat lung injury.

Method Twenty-three rat pubs were divided into four groups: airexposed control group $(\mathrm{n}=5)$, hyperoxia-exposed placebo group $(\mathrm{n}=7)$, hyperoxia-exposed BCG-vaccinated group $(\mathrm{n}=7)$, and air-exposed BCG-vaccinated group $(n=4)$. neonatal hyperoxic lung injury model was established according to the previous studies. Measurement of alveolar surface area, quantification of secondary crest formation, microvessel count, evaluation of alveolar septal fibrosis, and smooth muscle actin immunostaining were performed to assess hyperoxia-induced changes in lung morphology. The gene expression level was evaluated by RT-PCR.

Results The alveolar surface areas were significant different between the oxygen exposed placebo group and oxygen exposed BCG vaccinated group (alveolar surface area; $0.29 \pm 0.02 \mu \mathrm{m}^{2}$ and $0.52 \pm 0.04 \mu \mathrm{m}^{2} \mathrm{p}<0.05$ respectively). Number of crests and microvessel count was found to be significantly increased in the oxygen exposed BCG vaccinated group compared with the animals in the oxygen exposed placebo group $(\mathrm{p}<0.05)$. Exposure to hyperoxia resulted in a significant decrease in mean alveolar surface area and number of crests formed compared with air-exposed animals $(p<0.05)$. The degree of fibrosis was found to be significantly increased in the oxygen exposed placebo group compared with the animals in the oxygen exposed BCG vaccinated group (degree of fibrosis: $1.88 \pm 0.33$ and 0.91 $\pm 0.66 \mathrm{p}<0.05$ respectively). Immunostaining for SMA demonstrated hyperoxia-exposed animals with BCG vaccine in a significantly decrese in smooth muscle content compared with hyperoxia-exposed placebo animals $(\mathrm{p}<0.05)$. The expression of VEGF, FGF1, IL13, NF $\kappa$ B1 and TNF $\alpha$ in the lungs of vaccinated animals was significantly higher than that of non-vaccinated animals $(\mathrm{p}<0.05)$.

Conclusion Our results suggest that BCG vaccination can be a new protective strategy against neonatal hyperoxic lung injury.
These benefical effects may be interpreted with its immunomodulatory effects on proinflammatory-antiinflammatory cytocin balance and expression of growth factors.

\section{PS-210 ALVEOLAR CAPILLARY DYSPLASIA: A GENETICALLY DETERMINED DISRUPTION OF THE ALVEOLAR/ MESENCHYMAL CROSS-TALK CAUSING NEONATAL HYPOXIC FAILURE}

${ }^{1} \mathrm{C}$ Lizama, ${ }^{2} \mathrm{D}$ Peca, ${ }^{3} \mathrm{PE}$ Cogo, ${ }^{4}$ I Stucin-Gantar, ${ }^{5} \mathrm{P}$ Ursell, ${ }^{6} \mathrm{~A}$ Zovein, ${ }^{7} \mathrm{~A}$ VanHeijst, ${ }^{8} 0$ Danhaive. ${ }^{1}$ Cardiovascular Research Institute, University of California San Francisco, San Francisco, USA; 'Neonatology, Bambino Gesu Children's Hospital and Research Institute, Rome, Italy; ${ }^{3}$ Cardiology, Bambino Gesu Children's Hospital and Research Institute, Rome, Italy; ${ }^{4}$ Neonatology, University Medical Center, Ljubjana, Slovenia; ${ }^{5}$ Pathology, University of California San Francisco, San Francisco, USA; ${ }^{6}$ Neonatology, University of California San Francisco, San Francico, USA; ${ }^{7}$ Pediatrics, Radboud University Medical Center, Nijmegen, Netherlands; ${ }^{8}$ Pediatrics, University of California San Francisco, San Francisco, USA

\subsection{6/archdischild-2014-307384.508}

Background/aims Alveolar capillary dysplasia (ACD) is characterised by pulmonary veins misalignment, capillary paucity and alveolar misdevelopment, and caused by FOXF1 mutations only in 40\% of cases. Objectives were 1. to identify known and new gene defects and 2. to correlate them with molecular/cellular mechanisms.

Methods We recruited a cohort of 23 pathology-confirmed cases. When DNA was available, genome-wide copy number variation was analysed through Array Comparative Genomic Hybridization (aCGH). Mutations were tested by direct sequencing of FOXF1 and candidate genes identified by aCGH; Molecular pathways were analysed by multi-channel immunofluorescence microscopy of ACD cases compared to human fetal/neonatal lung tissue at various development stages.

Results 1. Genomic deletions or mutations were identified in $57 \%$ of tested cases. Besides FOXF1, two of the genes involved stand out as potential candidates: MEOX2 and TBX4.

2. ACD cases showed a markedly decreased expression of ckit, a marker expressed in pulmonary small arteries and capillaries in fetal lung controls. In normal fetal lungs FOXF1 and TBX4 were prevalently expressed at the mesenchymal-epithelial border, and MEOX2 in pulmonary vascular smooth muscle cells (PVSMC). Their expression pattern and intensity were altered in all ACD cases, indicating that decreased FOXF-1 and/or its downstream transcription factor TBX4 disrupt lung micro vessel formation and homing to alveolar epithelium, and that a similar phenotype may derive from dysregulated PVSMC proliferation and angiogenesis related to MEOX2 insufficiency.

Conclusion Genetic defects affecting the FOXF1 pathway affect the mesenchymal, endothelial and epithelial cross-talk leading to lung developmental disruption, pulmonary hypertension and hypoxic respiratory failure.

\section{PS-211 EARLY POSTNATAL SYSTEMIC LIPOPOLYSACCHARIDE INCREASES PRO-INFLAMMATORY CYTOKINES AND ANGIOGENIC GROWTH FACTORS IN THE LUNGS AND LEADS TO THE PHENOTYPE OF NEW BPD IN NEONATAL RATS}

${ }^{1} \mathrm{CW}$ Choi, ${ }^{2} \mathrm{JA}$ Lee, ${ }^{3}$ EK Kim, ${ }^{3} \mathrm{HS}$ Kim, ${ }^{4} \mathrm{BI}$ Kim, ${ }^{3} \mathrm{JH}$ Choi. ${ }^{1}$ Pediatrics, Seoul National University Bundnag Hospital, Seongnam, Korea; ${ }^{2}$ Pediatrics, Seoul National University Boramae Hospital, Seoul, Korea; ${ }^{3}$ Pediatrics, Seoul National University Hospital, Seoul, Korea; ${ }^{4}$ Pediatrics, Seoul National University Bundang Hospital, Seongnam, Korea

10.1136/archdischild-2014-307384.509 